\title{
Does volume matter? Incorporating estimated stone volume in a nomogram to predict ureteral stone passage
}

Nassib Abou Heidar'; Muhieddine Labban ${ }^{1}$; David-Dan Nguyen²; Adnan El-Achkar; Mazen Mansour ${ }^{1}$; Naeem Bhojani ${ }^{3}$; Rami Nasr ${ }^{1}$

${ }^{1}$ Division of Urology, Department of Surgery, American University of Beirut Medical Center, Beirut, Lebanon; ${ }^{2}$ Faculty of Medicine and Health Sciences, McGill University, Montreal, QC, Canada; ${ }^{3}$ Division of Urology, Department of Surgery, Centre hospitalier de l'Université de Montréal, Montreal, QC, Canada

Cite as: Heidar NA, Labban M, Nguyen D-D, et al. Does volume matter? Incorporating estimated stone volume in a nomogram to predict ureteral stone passage. Can Urol Assoc J 2021 October 18; Epub ahead of print. http://dx.doi.org/10.5489/cuaj.7364

Published online October 18, 2021

Corresponding author: Dr. Rami Nasr, American University of Beirut Medical Center, Beirut, Lebanon; rn05@aub.edu.lb

$* * *$

\section{Abstract}

Introduction: Recent studies have shown that software-generated 3D stone volume calculations are better predictors of stone burden than measured maximal axial stone diameter. However, no studies have assessed the role of formula estimated stone volume, a more practical and cheaper alternative to software calculations, to predict spontaneous stone passage (SSP).

Methods: We retrospectively included patients discharged from our emergency department on conservative treatment for ureteral stone $(\leq 10 \mathrm{~mm})$. We collected patient demographics, comorbidities, and laboratory tests. Using non-contrast computed tomography (CT) reports, stone width, length, and depth (w, l, d, respectively) were used to estimate stone volumes using the ellipsoid formula: $V=\pi^{*} 1^{*} \mathrm{w}^{*} \mathrm{~d}^{*} 0.167$. Using a backward conditional regression, two models were developed incorporating either estimated stone volume or maximal axial stone diameter. A receiver operator characteristic (ROC) curve was constructed and the area under the curve (AUC) was computed and compared to the other model.

Results: We included 450 patients; 243 patients (54\%) had SSP and 207 patients (46\%) failed SSP. The median calculated stone volume was significantly smaller among patients with SSP: $25(14-60) \mathrm{mm}^{3}$ vs. $113(66-180) \mathrm{mm}^{3}(\mathrm{p}<0.001)$. After adjusting for covariates, predictors of retained stone included: neutrophil to lymphocyte ratio (NLR) 
$\geq 3.14$ (odds ratio [OR] 6, $95 \%$ confidence interval [CI] 3.49-10.33), leukocyte esterase (LE) $>75$ (OR 4.83, 95\% CI 2.12-11.00), and proximal stone (OR 2.11, 95\% CI 1.16$3.83)$. For every $1 \mathrm{~mm}^{3}$ increase in stone volume, the risk of SSP failure increased by $2.5 \%$. The model explained $89.4 \%(0.864-0.923)$ of the variability in the outcome. This model was superior to the model including maximal axial diameter $(0.881,0.847-0.909$, $\mathrm{p}=0.04)$.

Conclusions: We present a nomogram incorporating stone volume to better predict SSP. Stone volume estimated using an ellipsoid formula can predict SSP better than maximal axial diameter.

\section{Introduction}

Kidney stones are a common disease affecting $8.8 \%$ of the United States' population; thereby, it is associated with considerable direct and indirect costs. ${ }^{1,2}$ The American Urological Association recommends conservative treatment using medical expulsive therapy (MET), alpha blockers, for uncomplicated distal ureteral stones $\leq 10 \mathrm{~mm}$. ${ }^{1}$ However, spontaneous stone passage (SSP) is dependent on the stone's size and ureteral position. ${ }^{3}$ Stone size is routinely measured using the maximum axial diameter on abdominal x-ray or computed tomography (CT) scan. ${ }^{4}$ Coll et al. showed that the SSP rate for stones between 2 and $4 \mathrm{~mm}$ is $76 \%$; for stones $5-7 \mathrm{~mm}, 60 \%$; for stones $7-9 \mathrm{~mm}$, $48 \%$; and only $25 \%$ for stones $>9 \mathrm{~mm}^{3}$ Accordingly, the maximal axial diameter captured on axial CT scan is classically used to estimate the stone burden and guide management. 1,5

While some studies argue that SSP is dependent on the stone's maximal axial diameter ${ }^{6,7}$, recent studies have suggested that an estimation of the stone volume is a more accurate predictor. ${ }^{8}$ Effectively, using 3D reconstruction software, stone volume could be estimated from CT scans. ${ }^{9}$ Nevertheless, these software are not readily available in all medical centers. ${ }^{10,11}$ Alternatively, the European Association of Urology suggests that a stone's length, width (axial diameter), and depth could be plugged into an ellipsoid formula to estimate the stone's volume. ${ }^{12}$ Moreover, Finch et al. demonstrated correlations between the gold standard 3D reconstructed volumes and volumes estimated using spheroid formulas. ${ }^{10}$

Most importantly, to minimize procedural morbidity, it is imperative to accurately identify which patients are in need of surgical intervention. ${ }^{13}$ Considering that no previously published nomogram included stone volume as a predictor of SSP ${ }^{14,15}$; we herein calculate the ellipsoid volume of stones to assess whether it predicts SSP. We will also compare the nomogram's performance to a nomogram incorporating the stone's maximal axial diameter instead of the volume. 


\section{Methods}

\section{Study population}

After institutional review board approval, electronic charts were queried for patients presenting for renal colic to our facility's emergency department (ED) between January 2010 and October 2018. We included only non-febrile patients with a single ureteral stone $\leq 10 \mathrm{~mm}$ in length, width, and depth diagnosed on non-contrast computed tomography (NCCT). Since SSP significantly decreases for larger stones, a $10 \mathrm{~mm}$ cutoff was chosen in concordance with contemporary series. ${ }^{16,17}$ We excluded patients with concurrent kidney or ureteral stones, patients with a solitary kidney, as well as patients with malignancies, evidence of infection, or chronic intake of steroids. In order to capture SSP, patients without a follow-up NCCT at four weeks of presentation were also excluded. Ureteral SSP was defined as absence of stone on follow-up NCCT scan. Based on regular practice in our ED, most patients were given MET using tamsulosin and analgesics.

\section{Data collection}

Information on patient demographics, basic metabolic profile, complete blood count with differential, urine analysis and culture, stone location, and stone length, width, and depth were collected. The NCCT scan results reported by a board-certified radiologist were used to identify stone location, size, and presence of hydronephrosis or fat streaking. Based on the Onen classification, hydronephrosis grades 0 and 1 were categorized as noto-mild hydronephrosis, a score of 2 was deemed as moderate hydronephrosis, and a score of 3 or 4 was considered as severe hydronephrosis.

\section{Volume estimation}

We gathered the length $(l)$, width $(w)$, and depth $(d)$ of stones. The optimal method to calculate stone volume is either through oblate (disk-like) or prolate (rugby) ellipsoid formulas because scalene ellipsoids (in which the lengths of all three stone dimensions are unequal) overestimate the volume of stones $<15 \mathrm{~mm} .{ }^{10}$ In fact, while stones $<9 \mathrm{~mm}$ in largest diameter trend towards a prolate shape, stones between 9 and $15 \mathrm{~mm}$ trend toward an oblate shape. ${ }^{18}$ Thus, stone volumes were estimated based on the following ellipsoid formula: $\pi^{*} 1^{*} \mathrm{w}^{*} \mathrm{~d} * 0.167$ where $l, w, d$ stand for length, width, and depth, respectively. $[12,18]$

\section{Covariates}

Data on patient demographics (gender and age), comorbidities (hypertension and diabetes), blood count (serum neutrophil-to-lymphocyte ratio, NLR), basic metabolic profile (creatinine), urine analysis (white blood cell count in urine and leukocyte esterase, LE) as well as radiological parameters (hydronephrosis, stone position, and stone size) 
were collected. We opted to include the neutrophil-to-lymphocyte ratio as it was demonstrated that a higher ratio is associated with retained stones. ${ }^{15,19}$

\section{Statistics}

We used independent t-test and chi-square test for continuous and categorical variables, respectively. Two models were constructed: (1) incorporating the calculated ellipsoid volume and (2) using the maximal axial diameter for the same cohort. Backward stepwise logistic regression was used to identify predictors of SSP, and the models were adjusted for the aforementioned covariates. Odds ratios and corresponding 95\% confidence intervals (CI) were obtained. Then, receiver operating characteristics (ROC) and associated areas under the curve (AUC) were calculated for each model. Then, DeLong et al.'s (1988) method was used to compare the areas under two ROC curves (paired design). ${ }^{20}$ Statistical tests were performed with a two-sided $p>0.05$ set for significance. All analyses were performed using SPSS version 24 (IBM Corp., Armonk, N.Y., USA) and MedCalc Statistical Software version 20.009 (MedCalc Software Ltd, Ostend, Belgium; https://www.medcalc.org; 2020).

\section{Results}

\section{Univariable predictors}

We included 450 patients; 243 patients (54\%) had SSP and 207 patients (46\%) had retained stone at follow up. Table 1 describes patient characteristics by stone passage status. The patients who experienced SSP were younger (median 41 years versus 47 years for patients with retained stones, $\mathrm{p}<0.001)$. Failure of stone passage was associated with proximal stones $(40.6 \%$ versus $17.3 \%)$, moderate-to-severe hydronephrosis $(51.6 \%$ versus $34.6 \%)$, and moderate-to-severe fat streaking (39.6\% versus $21.0 \%),(\mathrm{p}<0.001$ for all variables) (Table 1). Stone passage was associated with smaller width $(4.2 \pm 1.5$ mm vs. $6.2 \pm 1.6 \mathrm{~mm})$, smaller length $(3.9 \pm 1.2 \mathrm{~mm}$ versus $5.9 \pm 1.5 \mathrm{~mm})$, and smaller depth $(3.8 \pm 1.6$ versus $6.2 \pm 1.7 \mathrm{~mm})(\mathrm{p}<0.001$ for all variables $)$. Accordingly, the estimated average stone volume was also significantly smaller among patients with SSP: $25(14-60) \mathrm{mm}^{3}$ vs. $113(66-180) \mathrm{mm}^{3}, \mathrm{p}<0.001$. Figure 1 depicts how stone volume was inversely proportional to SSP irrespective of the stone's location.

\section{Adjusted predictors}

After adjusting for covariates, proximal stones had an odds ratio $[\mathrm{OR}=2.11 ; 95 \% \mathrm{CI}$ $(1.16-3.83)]$ for retained stones (Table 2). Moreover, NLR $\geq 3.14$ and LE $>75$ had $[6.00 ;(3.83-11.00)]$ and $[4.83 ;(2.12-11.00)]$ the odds for SSP failure, respectively. Furthermore, for every $1 \mathrm{~mm}^{3}$ increase in stone volume, the risk of SSP failure increased by $2.5 \% ;[1.025 ;(1.02-1.03)]$. 


\section{Area under receiver operating characteristic curve}

The nomogram including the calculated volume had an AUC 0.894; $(0.862-0.921)$ and the nomogram which incorporates the maximal axial diameter had an AUC 0.847 (0.847 - 0.909). A comparison of two ROC curves using a paired design revealed that volume was a better predictor of SSP than the maximal axial diameter (difference between areas $=0.0132 ;$ SE $0.000651 \mathrm{z}$ statistic $=2.033 ; \mathrm{p}<0.04)$.

\section{Discussion}

Although management strategies of ureteral stones range from conservative treatment to operative intervention, the literature and international guidelines lack individualized approaches to predict ureteral stone passage. ${ }^{5,21}$ Previous studies revealed that stone size, stone position, and elevated inflammatory markers are predictors of SSP. ${ }^{7,19,22,23}$ In this study, we assessed the role of a calculated ellipsoid volume reported on NCCT to predict SSP. We found serum NLR, urine leukocyte esterase, stone position, and stone volume to be predictors of SSP.

It is well established that stone size is a strong predictor of SSP; however, there is scarce evidence on the role of stone volume as a predictor of SSP. ${ }^{3,19,24,25} \mathrm{We}$ hypothesize that larger stone volumes would hinder stone passage due to a larger contact surface area, between the calculus and the ureter. ${ }^{26}$ Moreover, a larger stone volume means a theoretically heavier mass to be propelled through ureteral peristalsis. We believe that these factors explain the inversely proportional relationship between stone volume and SSP. To put it into perspective, a single millimeter increase in width from $5 \mathrm{x}$ $4 \times 3 \mathrm{~mm}(w \times l \times d)$ stone to a $6 \times 4 \times 3 \mathrm{~mm}$ yields an increase in volume from 31.46 $\mathrm{mm}^{3}$ to $37.76 \mathrm{~mm}^{3}$ translating to a $20.0 \%$ increase risk of retained stone.

SSP rates differ depending on whether the stone size is measured cranio-caudally (length) or axially (width). For instance, Tchey et al. reported an SSP rate approaches $89 \%$ for stones $<5 \mathrm{~mm}$ in length; whereas, a meta-analysis showed that stones $\leq 5 \mathrm{~mm}$ in width results in $68 \% \mathrm{SSP}{ }^{27,28}$ This demonstrates that a single size is not a good reflection of stone volume; thus, capturing the stone volume could enhance SSP prediction. $^{29}$

Computer-based algorithms have been devised to estimate ureteral stone burden and further improve the SSP prediction. Demehri et al. used a computer-based algorithm to demonstrate that the largest diameter estimate improves the accuracy of predicting SSP. ${ }^{30}$ A more elaborate algorithm developed by Jendeberg et al. uses 3D-segmentation to enhance ureteral stone passage prediction. ${ }^{31}$ These sophisticated programs, which rely on 3D reconstruction or computational algorithms, may be not accessible by the majority of physicians and radiologists. Therefore, we relied on Finch et al.'s demonstration of a reasonable correlation between software estimated volumes and formula-based estimates $(\mathrm{r}=0.77) .{ }^{10}$ According to our results, this method is reliable, cheaper, and a practical tool to be adopted by institutions. 
Our study is not without limitations. First, stone parameters were subject to systematic error because stone size relied on radiologists' measurement.. The authors acknowledge that this might not be a fully accurate representation of the true volume as stones could acquire different shapes dependent on the type of stone. ${ }^{18}$ Although stone type was not accounted for, we find that using the ellipsoid formula based on published evidence proves to be a quick and practical approach in ambulatory and emergency settings.

\section{Conclusions}

SSP prediction is of utmost importance in order to optimally counsel and manage patients presenting with ureteral stones. Herein, we demonstrate that stone volume, which could be easily estimated using a ellipsoid formula, has a predictive role in assessing SSP and was found to be superior than relying on maximal axial diameter alone. This nomogram could help guide the management of stone patients in the ED.

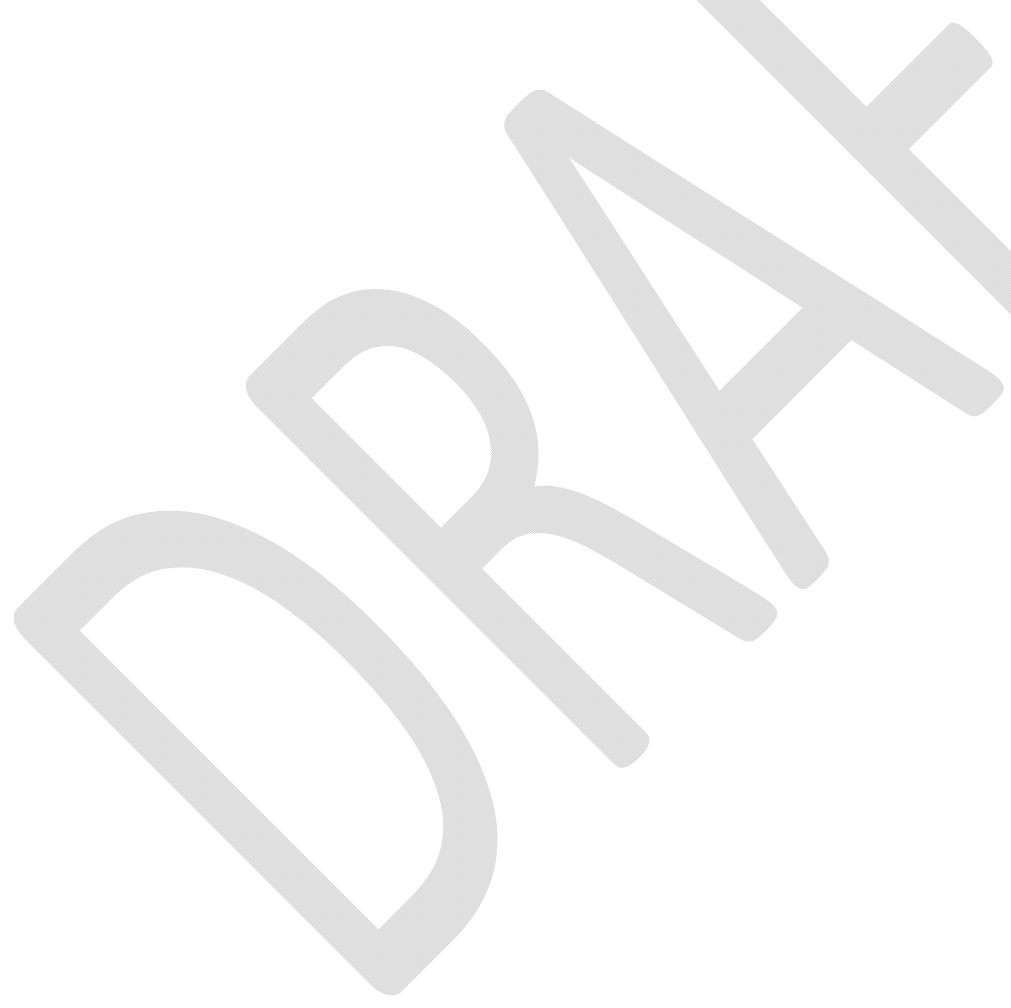




\section{References}

1. Assimos D, Krambeck A, Miller NL, et al. Surgical Management of Stones: American Urological Association/Endourological Society Guideline, PART I. Journal of Urology. 2016;196(4):1153-1160.

2. Saigal CS, Joyce G, Timilsina AR. Direct and indirect costs of nephrolithiasis in an employed population: opportunity for disease management? Kidney Int. 2005;68(4):1808-1814.

3. Coll DM, Varanelli MJ, Smith RC. Relationship of spontaneous passage of ureteral calculi to stone size and location as revealed by unenhanced helical CT. AJR American journal of roentgenology. 2002;178(1):101-103.

4. Ordon M, Andonian S, Blew B, Schuler T, Chew B, Pace KT. CUA Guideline: Management of ureteral calculi. Canadian Urological Association journal $=$ Journal de l'Association des urologues du Canada. 2015;9(11-12):E837-E851.

5. Türk C, Petř́k A, Sarica K, et al. EAU Guidelines on Diagnosis and Conservative Management of Urolithiasis. Eur Urol. 2016;69(3):468-474.

6. Skolarikos A, Laguna MP, Alivizatos G, Kural AR, de la Rosette JJ. The role for active monitoring in urinary stones: a systematic review. J Endourol. 2010;24(6):923-930.

7. Özcan C, Aydoğdu O, Senocak C, et al. Predictive Factors for Spontaneous Stone Passage and the Potential Role of Serum C-Reactive Protein in Patients with 4 to $10 \mathrm{~mm}$ Distal Ureteral Stones: A Prospective Clinical Study. J Urol. 2015;194(4):1009-1013.

8. Cui HW, Tan TK, Christiansen FE, Osther PJS, Turney BW. The utility of automated volume analysis of renal stones before and after shockwave lithotripsy treatment. Urolithiasis. 2020.

9. Jain R, Maskal S, Milk J, Kahn L, Fedrigon D, 3rd, Sivalingam S. Utility of stone volume estimated by software algorithm in predicting success of medical expulsive therapy. Can Urol Assoc J. 2021;15(3):E144-e147.

10. Finch W, Johnston R, Shaida N, Winterbottom A, Wiseman O. Measuring stone volume - 3D software reconstruction or an ellipsoid algebra formula? BJU international. $2013 ; 113$.

11. Wilhelm K, Miernik A, Hein S, et al. Validating Automated Kidney Stone Volumetry in CT and Mathematical Correlation with Estimated Stone Volume Based on Diameter. $J$ Endourol. 2018;32(7):659-664.

12. Tiselius HG, Ackermann D, Alken P, Buck C, Conort P, Gallucci M. Guidelines on urolithiasis. Eur Urol. 2001;40(4):362-371.

13. Lotan Y. Economics and cost of care of stone disease. Adv Chronic Kidney Dis. 2009;16(1):5-10.

14. Ganesan V, Kattan M, Loftus C, et al. MP01-02 A NOMOGRAM FOR PREDICTING URETERAL STONE PASSAGE. Journal of Urology. 2017;197(4S):e1-e1.

15. Abou Heidar N, Labban M, Najdi J, Al Shami A, Nasrallah O, Nasr R. Spontaneous ureteral stone passage: a novel and comprehensive nomogram. Minerva Urol Nefrol. 2021.

16. Selvi I, Baydilli N, Tokmak TT, Akinsal EC, Basar H. CT-related parameters and Framingham score as predictors of spontaneous passage of ureteral stones $\leq 10 \mathrm{~mm}$ : results from a prospective, observational, multicenter study. Urolithiasis. 2020. 
17. Cilesiz NC, Ozkan A, Kalkanli A, et al. Can serum procalcitonin levels be useful in predicting spontaneous ureteral stone passage? BMC Urol. 2020;20(1):42.

18. Finch W, Johnston R, Shaida N, Winterbottom A, Wiseman O. Measuring stone volume - three-dimensional software reconstruction or an ellipsoid algebra formula? BJU Int. 2014;113(4):610-614.

19. Abou Heidar N, Labban M, Bustros G, Nasr R. Inflammatory serum markers predicting spontaneous ureteral stone passage. Clin Exp Nephrol. 2020;24(3):277-283.

20. DeLong ER, DeLong DM, Clarke-Pearson DL. Comparing the Areas under Two or More Correlated Receiver Operating Characteristic Curves: A Nonparametric Approach. Biometrics. 1988;44(3):837-845.

21. Assimos D KA, Miller NL et al. Surgical management of stones: American Urological Association/Endourological Society Guideline, part II. Journal of Urology Web site. https://www.auanet.org/guidelines/kidney-stones-surgical-management-guideline. Published 2016. Accessed196: 1161.

22. Sfoungaristos S, Kavouras A, Katafigiotis I, Perimenis P. Role of white blood cell and neutrophil counts in predicting spontaneous stone passage in patients with renal colic. BJU Int. 2012;110(8 Pt B):E339-345.

23. Lee KS, Ha JS, Koo KC. Significance of Neutrophil-to-Lymphocyte Ratio as a Novel Indicator of Spontaneous Ureter Stone Passage. Yonsei Med J. 2017;58(5):988-993.

24. Shah TT, Gao C, Peters M, et al. Factors associated with spontaneous stone passage in a contemporary cohort of patients presenting with acute ureteric colic: results from the Multi-centre cohort study evaluating the role of Inflammatory Markers In patients presenting with acute ureteric Colic (MIMIC) study. BJU Int. 2019;124(3):504-513.

25. Sáenz Medina J, Alarcón Parra RO, Redondo González E, et al. [Prognostic factors of spontaneous expulsion in ureteral lithiasis]. Actas urologicas espanolas. 2010;34(10):882-887.

26. Lee SR, Jeon HG, Park DS, Choi YD. Longitudinal stone diameter on coronal reconstruction of computed tomography as a predictor of ureteral stone expulsion in medical expulsive therapy. Urology. 2012;80(4):784-789.

27. Preminger GM, Tiselius HG, Assimos DG, et al. 2007 Guideline for the management of ureteral calculi. Eur Urol. 2007;52(6):1610-1631.

28. Tchey DU, Ha YS, Kim WT, Yun SJ, Lee SC, Kim WJ. Expectant Management of Ureter Stones: Outcome and Clinical Factors of Spontaneous Passage in a Single Institution's Experience. Korean journal of urology. 2011;52(12):847-851.

29. Berkovitz N, Simanovsky N, Katz R, Salama S, Hiller N. Coronal reconstruction of unenhanced abdominal CT for correct ureteral stone size classification. European radiology. 2010;20(5):1047-1051.

30. Demehri S, Steigner ML, Sodickson AD, Houseman EA, Rybicki FJ, Silverman SG. CT-based determination of maximum ureteral stone area: a predictor of spontaneous passage. AJR American journal of roentgenology. 2012;198(3):603-608.

31. Jendeberg J, Geijer H, Alshamari M, Lidén M. Prediction of spontaneous ureteral stone passage: Automated 3D-measurements perform equal to radiologists, and linear measurements equal to volumetric. European radiology. 2018;28(6):2474-2483. 
Figures and Tables

Fig. 1. The rate of failure of stone passage by stone volume quartiles factored by ureteral location. SSP: spontaneous stone passage.

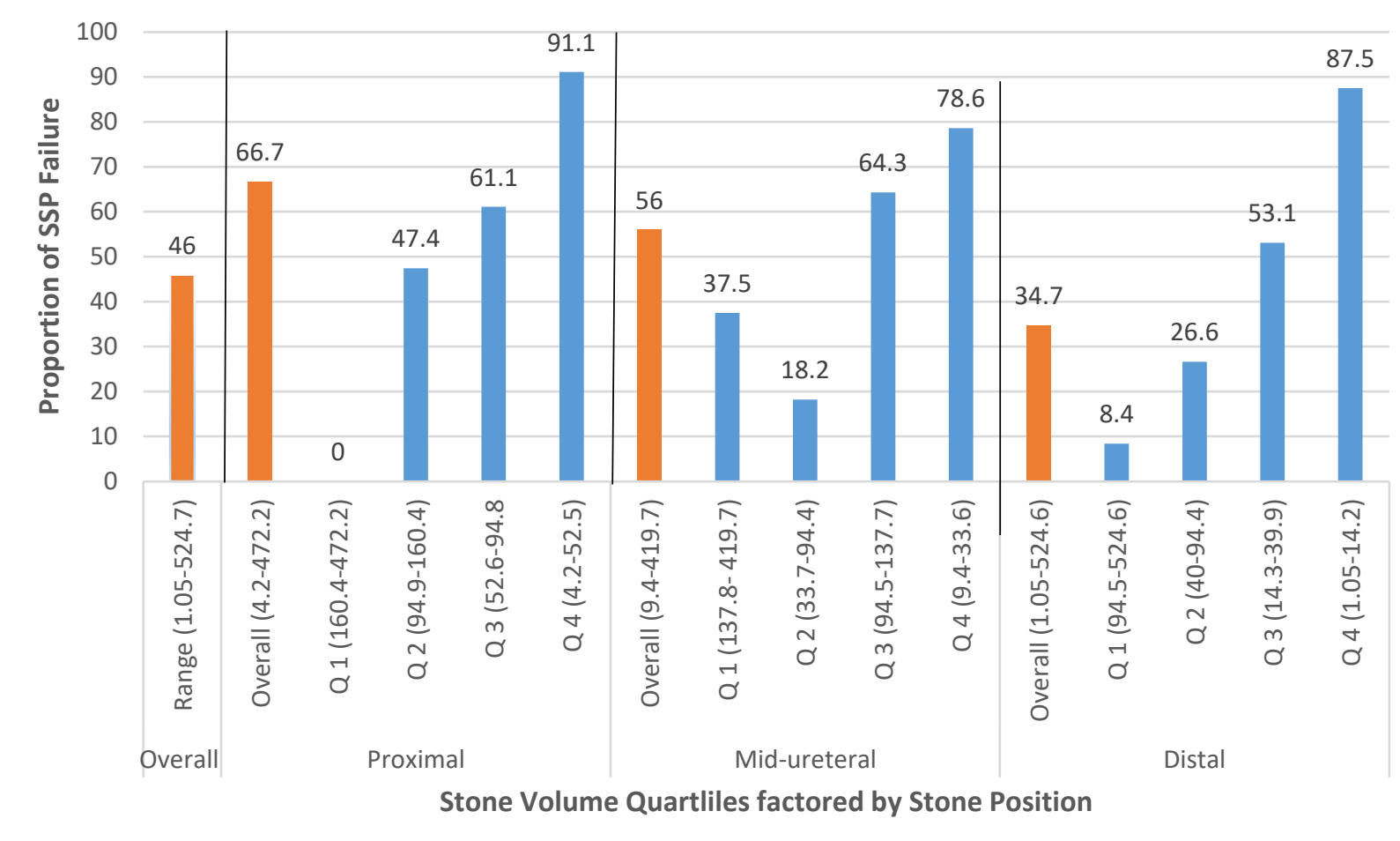


Table 1. Comparing the demographics, comorbidities, laboratory serum markers, and stone volumes among those who passed and failed to pass the ureteral stone using Student's t-test and Chi-squared

\begin{tabular}{|c|c|c|c|}
\hline Variable & $\begin{array}{l}\text { Failed SSP } \\
\qquad(n=207)\end{array}$ & $\begin{array}{c}\text { SSP } \\
(n=243)\end{array}$ & $\mathbf{p}$ \\
\hline & $\mathrm{n}(\%) /$ median (IQR) & $\mathrm{n}(\%) /$ median $(\mathrm{IQR})$ & \\
\hline Age & $47(37-57)$ & $41(32-52)$ & $<0.0001$ \\
\hline Female & $57(27.5 \%)$ & $53(21.8 \%)$ & 0.159 \\
\hline Hypertension & $71(34.3 \%)$ & $59(24.4 \%)$ & 0.02 \\
\hline Diabetes & $33(15.9 \%)$ & $25(10.3 \%)$ & 0.08 \\
\hline Creatinine & $1.1 \pm 0.4$ & $1.0 \pm 0.6$ & 0.03 \\
\hline $\mathrm{NLR} \geq 3.14$ & $141(68.1 \%)$ & $73(30.0 \%)$ & $<0.0001$ \\
\hline Hematuria & & & 0.9 \\
\hline 0-rare & $21(10.1 \%)$ & $20(8.2 \%)$ & \\
\hline 2-4 RBC/HPF & $15(7.2 \%)$ & $19(7.8 \%)$ & 8 \\
\hline 4-6 RBC/HPF & $14(6.8 \%)$ & $14(5.8 \%)$ & \\
\hline 8-10 RBC/HPF & $33(15.9 \%)$ & $44(18.1 \%)$ & \\
\hline Numerous RBC & $124(59.9 \%)$ & $146(60.1 \%)$ & \\
\hline Urine WBC & 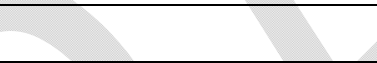 & 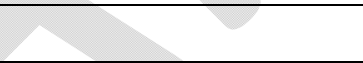 & \\
\hline 0-rare & $69(33.3 \%)$ & $116(47.7 \%)$ & $<0.0001$ \\
\hline 2-4 WBC/HPF & $61(29.5 \%)$ & $77(31.7 \%)$ & \\
\hline 4-6 WBC/HPF & $38(18.4 \%)$ & $16(6.6 \%)$ & \\
\hline 8-10 WBC/HPF & $14(6.8 \%)$ & $12(4.9 \%)$ & \\
\hline Numerous WBC & $25(12.1 \%)$ & $22(9.1 \%)$ & \\
\hline $\begin{array}{l}\text { Leukocyte esterase } \\
\geq 75\end{array}$ & $37(17.9 \%)$ & $16(6.7 \%)$ & $<0.0001$ \\
\hline Stone position & & & $<0.0001$ \\
\hline Proximal & $84(40.6 \%)$ & $42(17.3 \%)$ & \\
\hline Mid-ureteral & $28(13.5 \%)$ & $22(9.1 \%)$ & \\
\hline Distal & $96(45.9 \%)$ & $179(73.7 \%)$ & \\
\hline Fat streaking & & & $<0.0001$ \\
\hline None & $32(15.5 \%)$ & $77(31.7 \%)$ & \\
\hline Mild & $93(44.9 \%)$ & $115(47.3 \%)$ & \\
\hline Moderate & $63(30.4 \%)$ & $45(18.5 \%)$ & \\
\hline Severe & $19(9.2 \%)$ & $6(2.5 \%)$ & \\
\hline Hydronephrosis & & & $<0.0001$ \\
\hline None & $2(1.0 \%)$ & $21(8.6 \%)$ & \\
\hline
\end{tabular}




\begin{tabular}{|l|c|c|c|}
\hline Mild & $98(47.3 \%)$ & $138(56.8 \%)$ & \\
\hline Moderate & $92(44.4 \%)$ & $78(32.1 \%)$ & \\
\hline Severe & $15(7.2 \%)$ & $6(2.5 \%)$ & \\
\hline Stone width $(\mathrm{mm})$ & $6.2 \pm 1.6$ & $4.2 \pm 1.5$ & $<0.0001$ \\
\hline Stone length $(\mathrm{mm})$ & $5.9 \pm 1.5$ & $3.9 \pm 1.2$ & $<0.0001$ \\
\hline Stone depth $(\mathrm{mm})$ & $6.2 \pm 1.7$ & $3.8 \pm 1.6$ & $<0.0001$ \\
\hline $\begin{array}{l}\text { Ellipsoid volume } \\
\left(\mathrm{mm}^{3}\right)\end{array}$ & $113(66-180)$ & $25(14-60)$ & $<0.0001$ \\
\hline
\end{tabular}

HPF: high-power field; NLR: neutrophil to lymphocyte ratio; RBC: red blood cell count, SSP: spontaneous stone passage; WBC: white blood cell count.

\begin{tabular}{|l|c|c|}
\hline \multirow{2}{*}{$\begin{array}{l}\text { Table 2. Multivariable stepwise backward logistic regression predicting stone } \\
\text { passage for the nomogram incorporating calculated ellipsoid stone volume }\end{array}$} \\
\hline \multirow{2}{*}{ Variable } & \multicolumn{2}{|c|}{ SSP failure (n=436) } \\
\cline { 2 - 3 } NLR $\geq 3.14$ & Odds ratio (95\% CI) & p \\
\hline LE $>75$ & $4.83(2.12-11.00)$ & $<0.0001$ \\
\hline Proximal stone & $2.11(1.16-3.83)$ & $<0.0001$ \\
\hline Mid-ureteral stone & $1.24(0.54-2.89)$ & 0.015 \\
\hline Average ellipsoid & $1.025(1.02-1.03)$ & 0.614 \\
\hline
\end{tabular}

$95.3 \%$ of the patients were included in the multivariate analysis. Variables entered into the model: gender, creatinine, neutrophil-to-lymphocyte ratio (NLR), leukocyte esterase (LE), hydronephrosis, stone position, and calculated ellipsoid stone volume. CI: confidence interval; SSP: spontaneous stone passage. 